\title{
Treatment of atrial fibrillation in a district general hospital
}

\author{
Gregory Y H Lip, Kim Nyen Tean, Francis G Dunn
}

\begin{abstract}
Objective-To assess current strategies used to investigate and manage acute atrial fibrillation in hospital.

Design-Prospective survey of all acute admissions over 6 months.

Setting-District general hospital serving a population of 230000 in north east Glasgow.

Subjects-2686 patients admitted as emergency cases over 6 months.

Results-Of the 2686 patients, 170 (age range 38-95, mean (SD) $73.5(10 \cdot 6)$ years; 70 men (41\%) and 100 women $(59 \%)$ ) were admitted with atrial fibrillation. The principal underlying medical conditions were ischaemic heart disease in $\mathbf{7 9}$ $(46 \cdot 5 \%)$, rheumatic heart disease in 26 $(15 \cdot 3 \%)$, and thyroid disease in six $(3 \cdot 5 \%)$. Cardiac failure was present on admission in $61(36 \%)$, cerebrovascular events in $23(14 \%)$, and myocardial infarction in $17(10 \%)$. Of those with a history of atrial fibrillation (102 (60\%) including 10 with paroxysmal atrial fibrillation) treatment on admission included digoxin in $71(70 \%)$, warfarin in $20(20 \%)$, and aspirin in $17(17 \%)$; the aspirin was predominantly given for concomitant vascular disease. The mean (SD) inpatient stay was 16 days (19.7) (range 1-154) largely due to the patients with stroke. Thyroid function tests were performed in only $63 \%$ and echocardiography in 33\%. Overall, the rate of introduction of anticoagulation (seven patients) and attempted cardioversion (21 patients: 19 pharmacological and two electrical) was surprisingly low. Only 49 patients (34\% of those not on warfarin) had contraindications to anticoagulation: these included peptic ulcer or gastrointestinal bleeding in $18(12 \%)$, dementia in eight $(6 \%)$, chronic renal failure or dialysis in eight (6\%), and alcohol excess in four $(3 \%)$.

Conclusion-Standard investigations were inadequately used in patients with atrial fibrillation and there was a reluctance to perform cardioversion or to start anticoagulant treatment.
\end{abstract}

(Br Heart f 1994;71:92-95)

Atrial fibrillation is one of the most common cardiac arrhythmias among acute medical admissions to district general hospitals but there seems to be a wide range of views on its optimal management. This is not surprising as the management of the patient with atrial fibrillation is complicated by such things as diverse therapeutic options, concerns about anticoagulation, and the proarrhythmic effects of antiarrhythmic treatment. We report an audit relating to the investigation and management of patients admitted as emergencies with this dysrhythmia to a district general hospital, for a six month period between 1 September 1991 and 29 February 1992.

\section{Methods}

The hospital is a 990 bed district general hospital serving a population of 230000 in the north east part of the city of Glasgow and the borough of Strathkelvin. The hospital has a coronary care unit with six medical wards, a renal ward and a large geriatric department. There are nine general physicians, one of whom has a major interest in cardiology. Also there is a full time cardiologist, two renal physicians and four physicians with an interest in geriatric medicine, none of whom undertake general medical receiving duties. The physicians were informed at the start of the study that an audit was going to take place as this is part of hospital protocol. Thereafter, the information was collected from the case records and no further discussions were held with the physicians involved.

Patients were prospectively included in this study if they were found to be in established atrial fibrillation on the admission electrocardiogram, or had developed atrial fibrillation within 48 hours of admission. Patients were studied from the three medical units within the hospital, the coronary care unit the renal unit, and the geriatric wards.

The mode of presentation, cardiac investigations carried out, the aetiology of atrial fibrillation, the treatment before and that initiated after admission, and the inpatient mortality were also noted. The survey therefore assessed the characteristics of the inpatients admitted with atrial fibrillation, duration of stay, whether the aetiology of atrial fibrillation was assessed, what investigations were undertaken, and what their impact on management was and also actual management undertaken on the patient. This was done at the time of admission, during their stay in hospital, and at the time of discharge. 
Table 1 Demographic data

Acute medical (6 months) 2686

Admissions with

atrial

fibrillation $\quad 170(6.3 \%)$

Sex ratio

(men:women) 70:100

Age

(mean (SD)) $73.5(10.6)$

\section{Results}

During the six months of the study the total number of emergency admissions to the medical and geriatric services was 2686, an average of 448 patients/month (table 1). Over this period, 170 patients $(6.3 \%)$ were found to have atrial fibrillation (an average of 28 patients/month) of these, 100 (59\%) were women. Their mean (SD, range) age was $73.5(10.6,38-95)$ years. One hundred and two $(60 \%)$ patients had a history of atrial fibrillation (including $10(3.5 \%)$ who had had paroxysmal atrial fibrillation), and $68(40 \%)$ were newly diagnosed patients, with no previous record of the dysrhythmia.

MEDICAL HISTORY AND LIKELY UNDERLYING AETIOLOGY OF ATRIAL FIBRILLATION

Table 2 shows the underlying medical conditions in patients with atrial fibrillation. The principal conditions were ischaemic heart disease in $79(46.5 \%)$, hypertension in $30(17 \cdot 6 \%)$, rheumatic heart disease (predominantly mitral valve disease) in 26 $(15 \cdot 3 \%)$, and thyroid disease in six $(3.5 \%)$. Also, 38 patients $(22.4 \%)$ had a history of cerebrovascular disease, either a stroke or transient ischaemic attack.

\section{SYMPTOMS AND PRESENTING FEATURES ON} ADMISSION

The commonest presenting symptoms were typically cardiac (table 3). The principal symptoms were breathlessness in $88(51.8 \%)$, chest pain in $58(34 \cdot 1 \%)$, palpitation in 44 $(25.9 \%)$, and dizziness or syncope in 32 $(18 \cdot 8 \%)$

The predominant presenting clinical features included heart failure in $61(35.9 \%)$, a cerebrosvascular event in $23(13.5 \%)$, myocardial infarction in $17(10 \%)$, angina in $14(8 \cdot 2 \%)$, chest infection in $12(7 \cdot 1 \%)$, and digitoxicity in three $(1 \cdot 8 \%)$.

\section{INVESTIGATIONS}

All patients had an electrocardiogram and chest $x$ ray film on admission. Recording of

Table 2 Underlying medical conditions

\begin{tabular}{ll}
\hline & $\begin{array}{l}\text { Number(\%) } \\
\text { (total } n=170)\end{array}$ \\
\hline Ischaemic heart disease & $79(46 \cdot 5)$ \\
Hypertension & $30(17 \cdot 6)$ \\
Rheumatic heart disease & $26(15 \cdot 3)$ \\
Thyroid disease & $6(3 \cdot 5)$ \\
\hline
\end{tabular}

Table 3 Symptoms and presenting features on admission

\begin{tabular}{ll}
\hline & $\begin{array}{l}\text { Number(\%) } \\
\text { (total } n=170)\end{array}$ \\
\hline Symptoms: & \\
Dyspnoea & $88(51 \cdot 8)$ \\
Chest pain & $58(34 \cdot 1)$ \\
Palpitation & $44(25 \cdot 9)$ \\
Dizziness or syncope & $32(18 \cdot 8)$ \\
Presenting features: & \\
Heart failure & $61(35 \cdot 9)$ \\
Cerebrovascular event & $23(13 \cdot 5)$ \\
Myocardial infarction & $17(10)$ \\
Angina & $14(8 \cdot 2)$ \\
Chest infection & $12(7 \cdot 1)$ \\
Digitoxicity & $3(1 \cdot 8)$ \\
\hline
\end{tabular}

clinical data was variable, and complete information on investigations undertaken was only available for 146 patients $(86 \%$ of 170$)$. Echocardiography was performed in 48 patients $(33 \%)$. Cardiac enzymes and thyroid function tests were performed in 92 (63\%). A treadmill exercise test was performed in five patients $(3 \cdot 4 \%)$, and 24 hour Holter monitoring was performed in eight patients $(5 \cdot 5 \%)$.

In the 170 patients with atrial fibrillation, a new myocardial infarction was identified on the electrocardiogram in 12 patients $(7 \cdot 1 \%)$, and an old infarction was present in 15 patients $(8 \cdot 8 \%)$. The commonest was an inferior myocardial infarction, present in 17 (63\% of the 27 patients with an infarct on the electrocardiogram). Cardiac ischaemic changes were found in 29 patients $(17 \%$ of all admissions).

Chest $x$ rays films showed cardiomegaly in 93 patients $(54 \cdot 7 \%)$ of those admitted in atrial fibrillation. Pulmonary congestion was present in 77 patients $(45 \cdot 3 \%)$. Of the 48 patients in whom echocardiography was performed, findings included large left atria in $31(64.6 \%$ of 48$)$, mitral valve disease in 39 $(81 \cdot 3 \%)$, poor left ventricular function in 30 $(62.5 \%)$, and left ventricular hypertrophy in $11(22 \cdot 9 \%)$.

TREATMENT BEFORE ADMISSION

In those patients with a history of atrial fibrillation (102, including 10 with paroxysmal atrial fibrillation) the commonest treatment before admission was digoxin in $71(70 \%)$. A diuretic was given in $89(52.4 \%)$, and a $\beta$ blocker was used in $16(9.5 \%)$. Out of these 102 patients, antithrombotic treatment was used in only 37 patients, with $20(20 \%)$ given warfarin treatment and $17(17 \%)$ aspirin. Among the 17 patients on aspirin, 11 patients were taking aspirin for concomitant vascular disease rather than prophylaxis against thromboembolism.

TREATMENT INITIATED AFTER ADMISSION In the 68 patients $(40 \%$ of 170$)$ without a history of atrial fibrillation, digoxin was used in $51(75 \%)$ and amiodarone in $23(34 \%)$. Additional treatment started in the group as a whole included a $\beta$ blocker in six patients (3:5\% for rate control in atrial fibrillation and treatment of associated angina) and diuretics, with intravenous diuretic required on admission in 52 patients $(30.6 \%)$ and subsequent maintainence oral diuretic required in 46 patients $(27 \%)$. Antithrombotic treatment was started in 27 patients. Seven patients $(5 \%$ of the 150 not previously on warfarin) were given warfarin, and 20 (15\% of the 138 not previously on aspirin) were started on aspirin treatment. Of these 20 patients only five were given aspirin as prophylaxis against embolic events; the rest (15 patients) were given aspirin for concomitant vascular disease. Contraindications to anticoagulation were only identified in 49 patients (34\% of those not on warfarin); and these included peptic ulcer or gastrointestinal bleeding in 18 $(12 \%)$, dementia in eight $(6 \%)$, chronic renal 
failure or dialysis in eight (6\%), Crohn's disease in one $(0.6 \%)$, alcohol excess in four $(3 \%)$, and other major illness in $10(7 \%)$.

Cardioversion was attempted in 21 cases ( $12.4 \%$ of all admissions). Pharmacological cardioversion was the commonest method, used in 19 patients $(11.2 \%)$, particularly in the coronary care unit. Of these, 18 patients were given amiodarone, but one patient cardioverted after intravenous digoxin. Two patients did not cardiovert. The remaining two patients $(1 \cdot 2 \%)$ underwent direct current cardioversion.

\section{DURATION OF STAY AND INPATIENT MORTALITY}

Complete information on duration of stay was available for 146 patients (86\% of 170 ). The mean (SEM, range) inpatient stay was 16 (1.63, 1-154) days, largely due to patients with stroke. There was a weak but significant correlation between age and duration of stay (Spearman $r=0.22 ; \mathrm{p}<0.01$ ).

There were a total of $26(15.3 \%)$ inpatient deaths among our patients. The predominant causés of death were ischaemic heart disease and heart failure. No post mortem examinations were undertaken.

\section{Discussion}

The main reasons for treating atrial fibrillation are the prophylaxis of thromboembolic events and control of the ventricular rate. Treatment of atrial fibrillation also improves overall cardiac function and exercise tolerance in these patients. There is, however often wide variation in the management of this arrhythmia among physicians, both in the choice of investigations and on specific aspects of treatment-for example, in the initiation of anticoagulation. ${ }^{1}$

Standard investigations in a patient with atrial fibrillation should include an assessment of history, clinical state, and possible precipitating factors (alcohol, infection, and others), thyroid state by thyroid function tests, and an assessment of cardiac state by an electrocardiogram, chest $x$ ray film, and echocardiography: Echocardiography will provide useful information on cardiac function and structural heart disease in these patients. Also, measurement of cardiac enzymes (in an acute presentation) and 24 hour Holter monitoring may also be required.

Our study shows a suboptimal application of these standard investigations in the management of patients with atrial fibrillation. For example, echocardiography was only performed in $33 \%$ of patients. Interestingly, of these patients, $65 \%$ had dilated left atria, $81 \%$ had mitral valve disease, and $63 \%$ had poor left ventricular function, indicating that useful information was obtained from this investigation. Also, 24 hour Holter monitoring was performed in only $5.5 \%$ of patients, though $18.8 \%$ had a history of dizziness or syncope. This has important implications as ventricular standstill may commonly occur in patients with controlled atrial fibrillation who complain of dizziness or syncope, and most of these patients will benefit from permanent cardiac pacing. ${ }^{2}$ Another mandatory investigation, thyroid function tests, was performed in only $63 \%$. This also has important implications as atrial fibrillation commonly complicates hyperthyroidism, and clinically occult thyrotoxicosis may be the cause of idiopathic atrial fibrillation in an important proportion of cases. ${ }^{3}$

Though the commonest underlying pathology in our patients was ischaemic heart disease, ischaemic changes not associated with infarction were seen on the electrocardiogram in only $17 \%$ of patients, whereas changes due to infarction were present in $16 \%$ (the commonest was an inferior myocardial infarction). It has been previously noted, however, that when atrial fibrillation is associated with myocardial infarction it is important as an indicator of underlying ventricular dysfunction and a compromised myocardium, although there was no independent effect on mortality. ${ }^{4}$

The frequency with which heart failure accompanies atrial fibrillation is shown by our study. For example $52 \%$ of our patients presented with dyspnoea, $45 \%$ of patients had pulmonary congestion evident on the chest $x$ ray film, $63 \%$ of patients had poor left ventricular function on the echocardiogram, and intravenous diuretic was required acutely on admission in $31 \%$ of patients (with subsequent maintenance oral diuretic required in $27 \%$ ). Clinical heart failure was present in $36 \%$ of our patients. A similar proportion $(37.5 \%)$ of patients with atrial fibrillation was shown in a study of admissions with heart failure to a district general hospital. ${ }^{5}$ The presence of atrial fibrillation, however, may be an indicator for an increased risk of death, especially in patients with heart failure and lower filling pressures secondary to vasodilator or diuretic treatment. ${ }^{6}$

Recent large scale randomised studies have established the importance of anticoagulation treatment in the prophylaxis against thromboembolic events in patients with chronic atrial fibrillation..$^{7-12}$ When our study was performed only three of the five large scale studies had been published, but it was evident then that warfarin treatment reduced the risks of stroke by two thirds in patients with chronic atrial fibrillation..$^{7-10}$ Consensus about initiating oral anticoagulation treatment, however, continues to be lacking among general physicians as the rate of anticoagulation in our study was surprisingly low, with only $5 \%$ of patients having warfarin added to their treatment. The main concerns about starting warfarin seem to be the inconvenience and safety. ${ }^{13}$ Age, however, should not be a contraindication to initiating oral anticoagulation. ${ }^{10}$ The main reason for variation by physicians in initiation of such treatment seems to be the perceived differences in the estimated risk of systemic embolism in individual patients. ${ }^{1}$ Aspirin is thought to be an alternative and was started in 20 patients in this study (although in most this was for 
concomitant vascular disease rather than prophylaxis against thromboembolism), but in fact, only two of the five trials of warfarin in atrial fibrillation have reported a reduction (by about $25 \%$ ) of thromboembolic risk with aspirin. ${ }^{713}$ The use of aspirin alone, therefore, as an effective antithrombotic agent in atrial fibrillation remains controversial. ${ }^{13}$

The haemodynamic disturbance of atrial fibrillation results essentially from the absence of atrial systole and from the rapidity and irregularity of the ventricular response. With increasing age or heart failure, atrial systole contributes an increasing amount towards overall stroke volume. Indeed there is a significantly improved cardiac output and exercise capacity after cardioversion of atrial fibrillation to sinus rhythm. ${ }^{14}$ In our study, there was a low rate of attempted cardioversion ( $12 \%$ of patients), with pharmacological cardioversion the commonest method. For a patient whose rhythm is atrial fibrillation, the most complete relief of symptoms often occurs when sinus rhythm is restored. Cardioversion should therefore have been attempted in most patients who present with, atrial fibrillation of recent onset. The procedure is generally most successful in younger patients with no underlying cardiac disorder and has the added advantage that warfarin can be withdrawn after two to four weeks if sinus rhythm is maintained. ${ }^{15}$

This study establishes the importance of atrial fibrillation as a presenting dysrhythmia amongst emergency medical admissions in a Scottish district general hospital. As complete information on duration of stay and investigations undertaken was available in only $86 \%$ of our patients, our study also highlights some of the problems that occur in producing accurate figures for admissions. Our study provides useful information about a common condition and is particularly important at this time when medical audits are playing a larger part in planning the allocation of resources. For example, the mean inpatient stay in our study was 16 days, but with a wide range of 1-154 days. This finding and the significant correlation between age of patient and duration of inpatient stay has important implications as the general population becomes more elderly (with a greater life expectancy) and as the prevalence of atrial fibrillation increases with age from $0 \cdot 5 \%$ at $50-59$ to $8 \cdot 8 \%$ at $80-89 . .^{16-18}$

In conclusion, atrial fibrillation is a common finding among emergency medical admissions to a district general hospital.
Heart failure is the commonest presenting clinical feature, and ischaemic heart disease is the most frequent underlying cause. There is also a suboptimal application of standard investigations in patients with atrial fibrillation, and a reluctance to start oral anticoagulation treatment or to consider cardioversion, both of which are playing an increasingly prominent part in the management of this group of patients. These issues need to be considered to allow better management of this common arrhythmia.

1 Chang HJ, Bell JR, Deroo DB, Kirk JW, Wasson JH. Physician variation in anticoagulating patients with atrial fibrillation. Arch Intern Med 1990;150:81-4

2 Rebello R, Brownlee WC. Intermittent ventricular standstill during chronic atrial fibrillation in patients with dizziness or syncope. PACE 1987;10:1271-6.

3 Forfar JC, Miller HC, Toft AD. Occult thyrotoxicosis: correctable cause of 'idiopathic' atrial fibrillation. $A m \mathcal{F}$ Cardiol 1979;44:9-12.

4 Goldberg RJ, Seeley D, Becker RC, Brady P, Chen Z Osganian V, et al. Impact of atrial fibrillation on the inhospital and long-term survival of patients with acute myocardial infarction: a community-wide perspective. Am Heart f 1990;119:996-1001.

5 Parameshwar J, Poole-Wilson PA, Sutton GC. Heart failure in a district general hospital. $\mathcal{f} R$ Coll Physicians Lond 1992;26:139-42.

6 Middlekauff HR, Stevenson WG, Stevenson LW. Prognostic significance of atrial fibrillationin advanced heart failure. A study of 390 patients. Circulation heart failure.

7 Petersen P, Boysen G, Godtfredsen J, Andersen ED, Andersen B. Placebo controlled, randomised trial of warfarin and aspirin for prevention of thromboembolic warfarin and aspirin for prevention of thromboembolic
complications in chronic atrial fibrillation: the complications in chronic atrial fibrillation:
Copenhagen AFASAK study. Lancet 1989;1:175-9.

8 Stroke Prevention in Atrial Fibrillation Study Group Investigators. Preliminary report of the stroke prevention in atrial fibrillation study. $N$ Engl $\mathcal{F}$ Med 1990 322:863-8.

9 Stroke Prevention in Atrial Fibrillation Investigators Stroke prevention in atrial fibrillation study. Final results. Circulation 1991;84:527--39.

10 Boston Area Anticoagulation Trial for Atrial Fibrillation Investigators. The effect of low-dose warfarin on the risk of stroke in patients with non-rheumatic atrial fibrillation. N Engl f Med 1990;323:1505-11.

11 Connolly SJ, Laupacis A, Gent M, Roberts RS, Cairns JA, Joyner C. Canadian Atrial Fibrillation Anticoagulation (CAFA)Study. f Am Coll Cardiol 1991;18:349-55.

12 Ezekowitz MD, Bridgers SL, James KE, et al. Warfarin in the prevention of stroke associated with nonrheumatic the prevention of stroke associated with nonrheunat

13 Lowe GDO. Antithrombotic treatment and atrial fibrillation. $B M F$ 1992;305:1445-6.

14 Lipkin DP, Frenneaux M, Stewart R, Joshi J, Lowe T, et al. Delayed improvement in exercise capacity after cardioversion of atrial fibrillation to sinus rhythm. $\mathrm{Br} \mathrm{Hea}$ f 1988;59:572-7.

15 Dunn M, Alexander J, de Silva $R$, Hildner $F$ Antithrombotic therapy in atrial fibrillation. Ches 1989;95:118S-27S.

16 Wolf PA, Dawber TR, Thomas HE Jr, Kannel WB. Epidemiologic assessment of chronic atrial fibrillation and risk of stroke: the Framingham study. Neurology 1978;28:973-7.

17 Wolf PA, Kannel WB, McGee DL, Meeks SL, Bharucha NE, McNamara PM. Duration of atrial fibrillation and imminence of stroke: the Framingham study. Stroke 1983;14:664-7.

18 Wolf PA, Abbot RD, Kannell WB. Atrial fibrillation as an independent risk factor for stroke: the Framingham study. Stroke 1991;22:983-8. 